\title{
Frecuencia de infecciones quirúrgicas en pacientes del servicio de cirugía, Hospital II-1 Moyobamba, Perú, 2020
}

\section{Frequency of surgical infections in patients of the surgery service, Hospital II-1 Moyobamba, Peru, 2020}

\author{
Yaneliz Gómez Santiago ${ }^{1}$, Yshoner Antonio Silva Díaz ${ }^{2}$
}

\section{RESUMEN}

El objetivo del estudio fue determinar la frecuencia de infecciones quirúrgicas en pacientes del servicio de Cirugía del Hospital II-1 de la Provincia de Moyobamba, departamento de San Martín, Perú, en el período 2020. El estudio fue de nivel descriptivo. La muestra estuvo constituida por 51 historias clínicas de pacientes postoperados siguiendo un muestreo no probabilístico por conveniencia, el instrumento aplicado fue una ficha de registro de datos sobre las infecciones quirúrgicas y datos relacionados. Los resultados evidencian que del $100 \%$ (51) de pacientes, el $35.3 \%$ (18) tuvo herida infectada y el 64.7\% (33) tuvo herida no infectada, existiendo una frecuencia considerable de infecciones de herida quirúrgica $(35.3 \%)$ en el Servicio de Cirugía del Hospital II-1 de Moyobamba. Asimismo, las condiciones que influyeron para una mayor frecuencia de infecciones quirúrgicas fueron la duración prolongada de la cirugía y el rasurado de la zona operatoria $(p<0.05)$. Las condiciones que no influyeron fueron: la edad, sexo, tipo de intervención, diabetes, obesidad y profilaxis antibiótica no dada $(\mathrm{p}>0.05)$. Se concluye que existe una alta frecuencia de infecciones de herida quirúrgica en los pacientes del Servicio de Cirugía del Hospital II-1 de Moyobamba.

Palabras clave: Infección quirúrgica, herida operatoria

\begin{abstract}
The objective of the study was to determine the frequency of surgical infections in patients from the Surgery Service of Hospital II-1 of the Province of Moyobamba, department of San Martín, Peru, in the period 2020. The study was descriptive. The sample consisted of 51 clinical records of postoperative patients following a nonprobabilistic sampling for convenience, the instrument applied was a data record sheet on surgical infections and related data. The results show that of $100 \%(51)$ of patients, $35.3 \%(18)$ had an infected wound and $64.7 \%(33)$ had an uninfected wound, with a considerable frequency of surgical wound infections $(35.3 \%)$ in the Service of Surgery of Hospital II-1 of Moyobamba. Likewise, the conditions that influenced a higher frequency of surgical infections were the prolonged duration of surgery and the shaving of the operative area $(\mathrm{p}<0.05)$. The conditions that did not influence were: age, sex, type of intervention, diabetes, obesity and antibiotic prophylaxis not given $(p>0.05)$. It is concluded that there is a high frequency of surgical wound infections in patients of the Surgery Service of Hospital II-1 of Moyobamba.
\end{abstract}

Keywords: Surgical infection, operative wound

'Bachiller de la Escuela Profesional de Enfermería. Universidad Nacional Toribio Rodríguez de Mendoza de Amazonas. Correo electrónico: gosaya8@hotmail.com.

${ }^{2}$ Docente en la Escuela Profesional de Enfermería de la Facultad de Ciencias de la Salud de la Universidad Nacional Toribio Rodríguez de Mendoza de Amazonas.Correo electrónico: yshoner.silva@untrm.edu.pe. 


\section{INTRODUCCIÓN}

Las infecciones quirúrgicas son uno de los problemas frecuentes en el ámbito hospitalario, por lo tanto, deben ser abordados con la importancia del caso, puesto que son eventos que afectan la salud de las personas, generan secuelas, y ponen en tela de juicio las habilidades y capacidades de los médicos responsables. Aunque muchos prefieren señalar que las infecciones quirúrgicas se deben a la proliferación de agentes patógenos en el postoperatorio dejando de lado los factores subyacentes (Vásquez, 2016, p.6).

Una cirugía predispone una infección por la naturaleza del mismo procedimiento, puesto que en el proceso se alteran y rompen las primeras líneas de defensa del organismo: las barreras de la piel y mucosas. Por ello, al no tener un procedimiento quirúrgico seguro, el paciente puede sufrir una infección del sitio quirúrgico. En la actualidad, las infecciones quirúrgicas constituyen una causa frecuente de enfermedad y muerte en personas postoperadas (Tite, 2012, p.13). El origen de una infección quirúrgica tiene muchas causas y factores, que se agrupan en las siguientes dimensiones: los factores que dependen del germen, del paciente y los relacionados con la intervención y el ambiente hospitalario donde se realiza la intervención (Fernández, 2016,p.32).

Con respecto a los factores específicos que implican un riesgo para desarrollar una infección quirúrgica se encuentran los relacionados con el huésped (infección previa del sitio operatorio, sistema inmune deprimido, patologías crónicodegenerativas preexistentes, problemas de malnutrición y obesidad), los relacionados con la cirugía (la técnica inadecuada, la duración prolongada del acto quirúrgico, inadecuado lavado de manos, rasurado de la zona operatoria, las condiciones del quirófano, el uso profiláctico de antibióticos, entre otros) (Tite, 2012, p.13).

Un reporte de la Organización Mundial de la Salud (OMS) señalo que las infecciones quirúrgicas representan el $25 \%$ de todas infecciones hospitalarias, representando un porcentaje alarmante que debe ser abordado por los sistemas de salud con la finalidad de controlar este problema, y consecuentemente los efectos en los pacientes quienes sufren secuelas considerables tanto en su salud como en su recuperación para retornar a sus actividades productivas (Bernal, 2014, p.43). Asimismo, la infección de la herida operatoria constituye la tercera infección hospitalaria más frecuente y la primera, entre los pacientes que se someten a una cirugía (38 \%) (Tite, 2012, p.18). Por ello la OMS estableció "La seguridad de las prácticas quirúrgicas" como reto mundial para la seguridad del paciente en la salud. Dentro de este reto se aborda el manejo y la prevención de las infecciones quirúrgicas.

Otras evidencias recogidas por el Centro para el Control y Prevención de Enfermedades de Atlanta en diversos nosocomios de Estados Unidos señalan que el $5 \%$ de pacientes dados de alta en el mundo ha tenido una infección quirúrgica. Además, este problema genera, a parte del aumento de morbilidad y mortalidad, una estadía hospitalaria prolongada e incremento de los costos económicos y sociales (Kirby, 2018, p.3). Por otro lado, en Brasil, las infecciones quirúrgicas representan entre el 1,4\% y $38,8 \%$ del total de pacientes operados, encontrándose dentro del índice que hay a nivel de Latinoamérica, donde se calcula que, de cada 100 pacientes, 15 desarrollaron infección del sitio quirúrgico (Ángeles, 2014, p.8).

En el caso del Perú, un reporte sobre infecciones hospitalarias en nosocomios públicos señala que la incidencia de infecciones quirúrgicas es de $2.2 \%$ a $6.3 \%$ por cada 100 cirugías. También se señala que existe un alto índice de subregistro de estos problemas, puesto que en los hospitales existe un débil sistema de reporte e información, además muchos profesionales de la salud evitan reportar estos problemas para evitar problemas legales (Minsa, 2018, p.4). A pesar que el Ministerio de Salud ha implementado desde 1998 el "Protocolo: Estudio de Prevalencia de Infecciones Intrahospitalarias" y el Manual de Prevención y Control de las infecciones intrahospitalarias, el problema no se ha controlado y continúa siendo un problema incidente en los servicios de cirugía (Méndez, 2018,p.4).

En el Hospital de Moyobamba del departamento de San Martín, no se ha desarrollado una estrategia específica de reporte de infecciones quirúrgicas, menos de acciones institucionales para la prevención de las infecciones y el control de sus factores. La información con la que se cuenta proviene de las historias clínicas y de las experiencias de los profesionales de la salud del servicio de Quirófano y Cirugía donde según referencias de los mismos, existe un número considerable de infecciones quirúrgicas causadas por las condiciones en las que se realiza la cirugía en el hospital.

Respecto al Servicio de Cirugía del Hospital de Moyobamba, no se cuenta con un diagnóstico específico de infecciones de herida operatoria en los pacientes, tampoco de estudios e investigaciones que se hayan realizado en ese ámbito específico; sin embargo, de acuerdo a las referencias de los profesionales que ahí laboran hay varios pacientes que se complican con infecciones postoperatorias y ello alarga su estancia y recuperación. 


\section{MATERIAL Y MÉTODO}

El diseño de estudio fue no experimental descriptivo. La muestra estuvo constituida por 51 historias clínicas de pacientes postoperados entre los meses de enero y febrero del año 2020 en el servicio de cirugía del Hospital de Moyobamba. El método de investigación utilizado fue el método hipotético deductivo, y el método de recolección de datos fue la revisión documentaria. Excluyéndose las historias clínicas de pacientes que hayan tenido complicaciones graves en la intervención quirúrgica, que signifique un sesgo en el estudio, asimismo historias clínicas incompletas.

El instrumento utilizado fue una ficha de registro de datos sobre las infecciones quirúrgicas y datos relacionados al problema como los datos relacionados al paciente, el tipo de herida operatoria, el tipo de patología quirúrgica y datos relacionados a la intervención quirúrgica. Todos los ítems fueron planteados considerando una escala dicotómica.

\section{RESULTADOS}

\section{Figura 1}

Frecuencia de infección quirúrgica en pacientes del Servicio de Cirugía, Hospital II-1 Moyobamba.

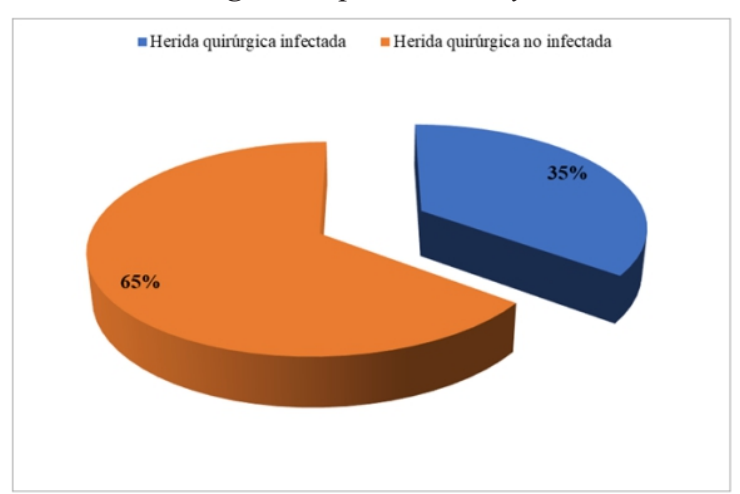

En la figura 1 se observa que del $100 \%$ (51) de pacientes, el $35.3 \%$ (18) tuvo herida infectada y el $64.7 \%$ (33) tuvo herida no infectada.

\section{Figura 2}

Frecuencia de infección quirúrgica según edad en pacientes del Servicio de Cirugía, Hospital II-1 Moyobamba.

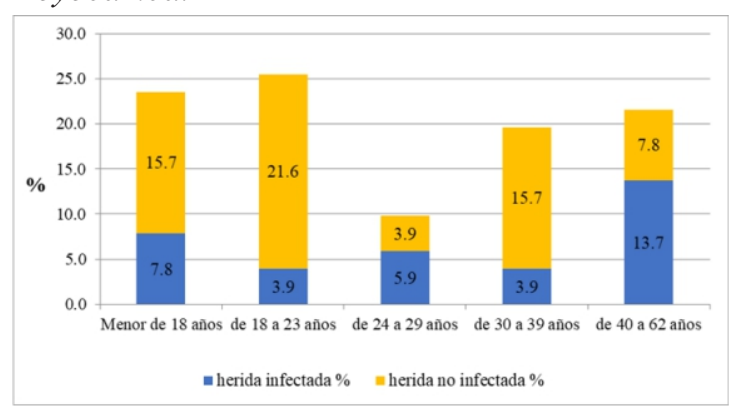

En figura 2 se muestra que del $100 \%$ (51) de pacientes, el $21.6 \%$ (11) tiene entre 18 a 23 años y al mismo tiempo herida no infectad, asimismo el $15.7 \%$ (8) tuvo entre 30 a 39 años y al mismo tiempo herida no infectada. El análisis estadístico indica que la edad no influyó en la frecuencia de infección de la herida operatoria $(\mathrm{p}>0.05)$.

\section{Figura 3}

Frecuencia de infección quirúrgica según sexo en pacientes del Servicio de Cirugía, Hospital II-1 Moyobamba

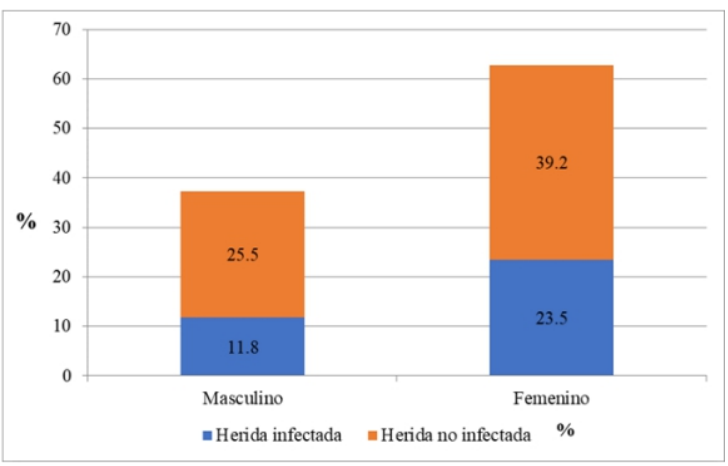

En la figura 3, se evidencia que del $100 \%$ (51) de pacientes, el $39.2 \%$ (20) es de sexo femenino y tiene herida no infectada, asimismo el $25 \%$ (13) es de sexo masculino y no tiene herida infectada. El análisis estadístico indica que el sexo no influyó en la frecuencia de infección de la herida operatoria $(\mathrm{p}>0.05)$.

\section{Figura 4}

Frecuencia de infección quirúrgica según tipo de intervención en pacientes del Servicio de Cirugía, Hospital II-1 Moyobamba.

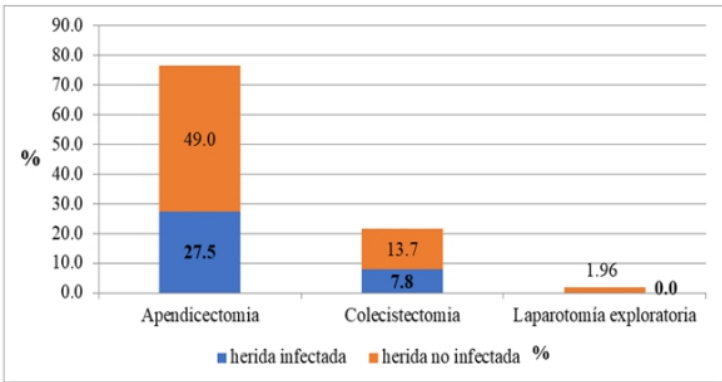

En la figura 4, se aprecia que del $100 \%$ (51) de pacientes, el $49 \%$ (25) fue operado de apendicectomía y tiene herida no infectada, asimismo el $27.5 \%$ (14) fue operado de apendicectomía y tiene herida infectada. Por otro lado, el $13.7 \%$ (7) fue operado de colecistectomía y tiene herida no infectada, asimismo el $7.8 \%$ (4) presenta herida infectada y fue operado de colescistectomía. Solo el $1.96 \%$ tuvo laparotomía exploratoria. El análisis estadístico indica que el tipo 
de operación no influyó en la frecuencia de infección de la herida operatoria $(\mathrm{p}>0.05)$.

\section{Figura 5}

Frecuencia de infección quirúrgica según condición de diabetes en pacientes del Servicio de Cirugía, Hospital II-1 Moyobamba.

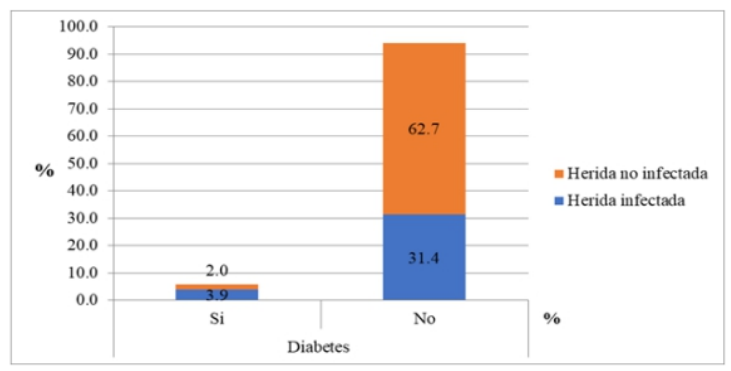

En la figura 5, se observa que del $100 \%$ (51) de pacientes, el $62.7 \%$ (32) no presentó diabetes y no tuvo herida infectada, sin embargo el $31.4 \%$ (16) no tuvo diabetes pero si tuvo herida infectada. Por otro lado el $3.9 \%$ (2) tuvo diabetes y herida infectada. El análisis estadístico indica que la diabetes no influyó en la frecuencia de infección de la herida operatoria $(\mathrm{p}>0.05)$.

\section{Figura 6}

Frecuencia de infección quirúrgica según condición de obesidad en pacientes del Servicio de Cirugía, Hospital II-1 Moyobamba.

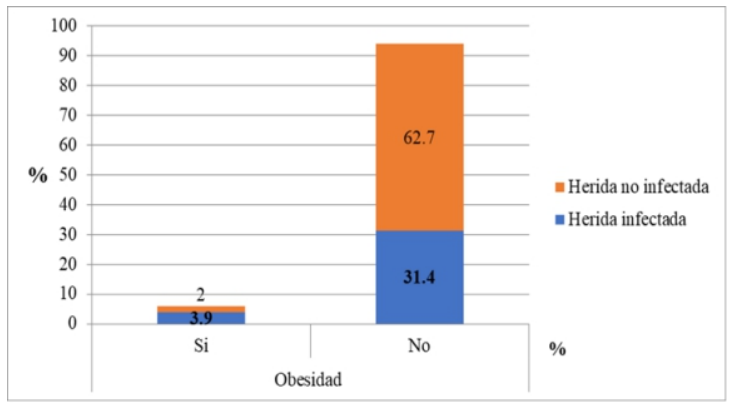

En la figura 6, se muestra que del $100 \%$ (51) de pacientes, el $62.7 \%$ (32) no presentó obesidad y no tuvo herida infectada, sin embargo, el $31.4 \%$ (16) no tuvo obesidad, pero sí tuvo herida infectada. Por otro lado, el 3.9\% (2) tuvo obesidad y herida infectada al mismo tiempo. El análisis estadístico indica que la obesidad no influyó en la frecuencia de infección de la herida $(\mathrm{p}>0.05)$.

\section{Figura 7}

Frecuencia de infección quirúrgica según condición de cáncer, Hospital II-1 Moyobamba.

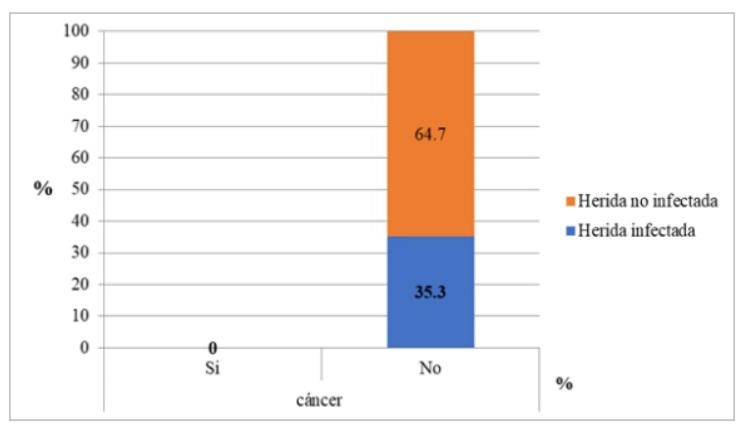

En la figura 7, se observa que del $100 \%$ (51) de pacientes, ningún paciente $(0 \%)$ presentó cáncer, por tanto, el análisis estadístico indica que no se puede determinar que si el cáncer influyó o no en la frecuencia de infección de la herida operatoria.

\section{Figura 8}

Frecuencia de infección quirúrgica según condición de malnutrición, Hospital II-1 Moyobamba.

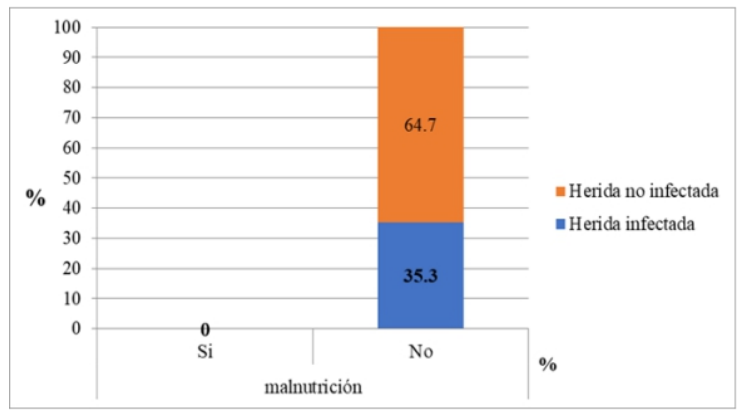

En la figura 8, se observa que del $100 \%$ (51) de pacientes, ningún paciente $(0 \%)$ presentó malnutrición, por tanto, el análisis estadístico indica que no se puede determinar que si la malnutrición influyó o no en la frecuencia de infección de la herida operatoria.

\section{Figura 9}

Frecuencia de infección quirúrgica según duración de la intervención. Hospital II-1 Moyobamba.

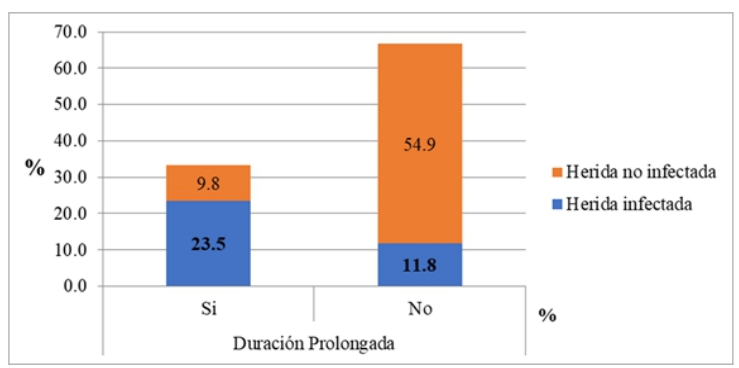

En la figura 9, se observa que del $100 \%(51)$ de pacientes, el $54.9 \%$ (28) tuvo una operación de duración prolongada y no presentó herida infectada; 
sin embargo, el $23.5 \%$ (12) tuvo una intervención prolongada y al mismo tiempo herida infectada. El análisis estadístico indica que la duración prolongada de la operación influyó en la frecuencia de infección de la herida operatoria $(\mathrm{p}>0.05)$.

\section{Figura 10}

Frecuencia de infección quirúrgica según rasurado de lazona operatorial.

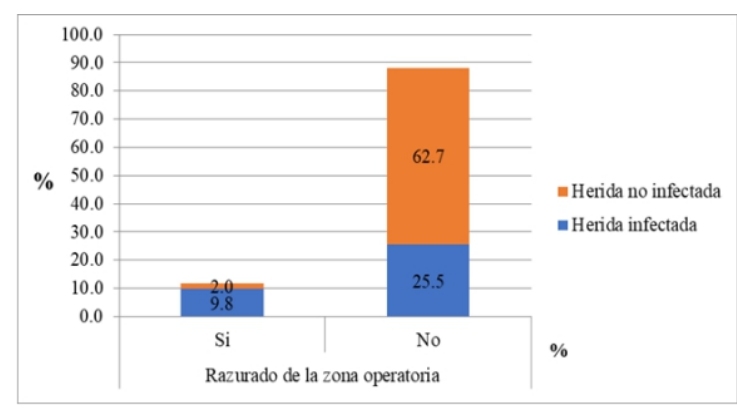

En la figura 10 se observa que del $100 \%$ (51) de pacientes, el $62.7 \%$ (32) fue rasurado en la zona operatoria y no presentó herida infectada; sin embargo, el $9.8 \%$ (5) fue rasurado y tuvo herida infectada. El análisis estadístico indica el rasurado de la zona operatoria influyó en la frecuencia de infección de la herida operatoria $(\mathrm{p}>0.05)$.

\section{Figura 11}

Frecuencia de infección quirúrgica según profilaxis antibiótica.

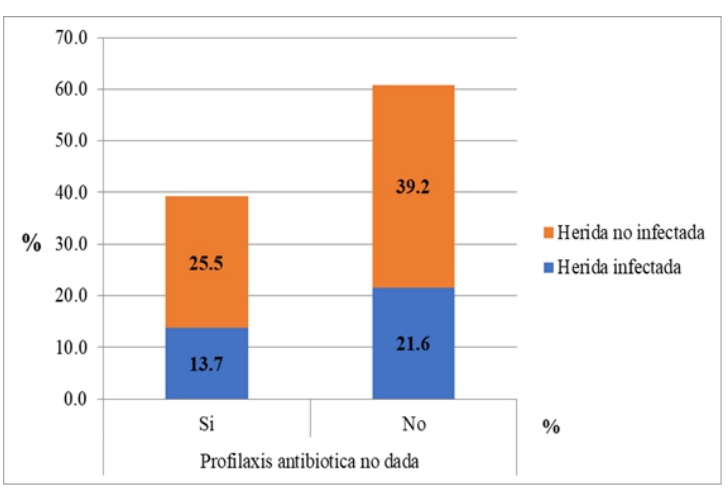

En la figura 11 se observa que del $100 \%$ (51) de pacientes, el $39.2 \%$ (20) recibió profilaxis antibiótica y no presentó herida infectada; sinembargo el $13.7 \%$ (7) no recibió profilaxis antibiótica y tuvo herida infectada. El análisis estadístico indica la profilaxis antibiótica no dada no influyó en la frecuencia de infección de la herida operatoria $(\mathrm{p}>0.05)$.

\section{DISCUSIÓN}

En los resultados del estudio se pudo encontrar una frecuencia importante de infecciones de herida quirúrgica (35.3\%) en los pacientes del Servicio de Cirugía, Hospital II-1 Moyobamba. Asimismo, las condiciones que influyeron para una mayor frecuencia de infecciones quirúrgicas fueron la duración prolongada de la cirugía y el rasurado de la zona operatoria $(p<0.05)$ y las condiciones que no influyeron significativamente fueron: la edad, sexo, tipo de intervención, diabetes, obesidad y profilaxis antibiótica no dada ( $\mathrm{p}>0.05)$.

Similares resultados encontraron De La Cruz (2015) en Lima, Rodríguez et al (2017) en Brasil, Carvajal \& Londoño (2011) en Colombia, Falci et al (2011) en Brasil, Vásquez et al. (2016) en Ecuador, Medina et al (2011) en España, Bernal (2014) en Cusco, Alvia (2012) en Lima, Casado (2014) en España y Fernández et al. (2016) en Cuba.

En cuanto a la frecuencia de infecciones quirúrgicas y sus factores relacionados, similares resultados encontraron De La Cruz (2015) en un estudio realizado en Lima, donde pudo evidenciar una frecuencia mayor al $20 \%$ de infecciones quirúrgicas y las variables asociadas fueron comorbilidad (OR: 4,7), Tiempo de cirugía $>1$ hora (OR: 5,2). Estos resultados concuerdan con los encontrados en el presente estudio respecto a la frecuencia de infecciones respiratorias y al factor duración prolongada de la cirugía.

Por otra parte, Rodríguez et al (2017) en una investigación realizada en Brasil evidenció que existe una frecuencia considerable de infecciones quirúrgicas (alrededor de $10 \%$ ) y los factores condicionantes son: tiempo de periodo preoperatorio mayor a 1 día; duración prolongada de la cirugía; características y contaminación de la herida operatoria o cuando la herida se clasifica en potencialmente contaminada, contaminada e infectada. Este estudio concuerda con los hallazgos del presente estudio en cuanto a la frecuencia de infecciones quirúrgicas y al factor duración prolongada de la cirugía

De igual manera Carvajal \& Londoño (2011) en Colombia también encontraron alta frecuencia de infecciones quirúrgicas (mayor al $15 \%$ ), además evidenciaron que la duración prolongada de la intervención, la inadecuada asepsia de la piel, el poco cuidado en el lavado de manos, la urgencia de intervención, la ventilación deficiente en el quirófano, presencia de drenajes, pobre hemostasia son factores que predisponen la infección de la herida operatoria. Esta investigación se asemeja al presente estudio en cuanto a los hallazgos sobre la frecuencia de infecciones quirúrgicas y el factor tiempo prolongado de cirugía.

Otro estudio donde se encontró similares resultados fue el de Falci et al (2011) en Brasil encontró que existen casos frecuentes de infecciones quirúrgicas en los hospitales de este país, encontrando como 
variables asociadas el nivel de contaminación del sitio operatorio, el tipo de intervención y el tiempo de duración de la cirugía, las cuales se asociaron estadísticamente $(\mathrm{p}<0.05)$ como factores de riesgo.

Asimismo, Vásquez et al (2016) también en Ecuador evidenció que la frecuencia de casos de infecciones quirúrgicas tiene como factores condicionantes aspectos relacionados con el quehacer médico y las condiciones del hospital y el servicio donde se realiza la intervención, asimismo los procedimientos previos que se dan en la cirugía, siendo estos resultados similares en cuanto aspectos relacionados con la intervención (duración prolongada de la cirugía y el rasurado de la zona operatoria).

Por otro lado, la investigación de Medina et al (2011) en España pudo evidenciar que existe un porcentaje importante de infecciones quirúrgicas, asimismo encontró que existe una asociación de riesgo entre el rasurado de la zona operatoria y el desarrollo de la infección del sitio quirúrgico. También Richter (2016) en Lima afirma, respecto a la frecuencia de infecciones quirúrgicas, que la duración prolongada de la cirugía es factor de riesgo para adquirir una infección quirúrgica. Estos resultados concuerdan con los encontrados en el presente estudio

Otro estudio realizado por Bernal (2014) en Cusco encontró una frecuencia elevada de infección quirúrgicas (mayor al $15 \%$ ), afirmando que la cirugía con pocas condiciones de asepsia y en una herida contaminada es un factor que facilita las complicaciones infecciosas en el postoperatorio. Esta investigación también encontró frecuencia elevada de infecciones quirúrgicas al igual que el presente estudio.

Otra investigación realizada por Alvia (2012) en Lima también pudo evidenciar una incidencia media de infecciones quirúrgicas en pacientes operados en general, además encontró que como factor de riesgo de la frecuencia de estas infecciones esta principalmente la presencia de herida sucia y contaminada en los pacientes que se sometieron a una intervención quirúrgica. En este estudio se encontraron otras variables asociadas con la infección quirúrgica; sin embargo, los resultados son semejantes en cuanto a la frecuencia de este tipo de infecciones.

De igual manera Casado (2014) en un estudio realizado en España encontró que, ante la frecuencia de infecciones quirúrgicas, el uso de la utilización de antibióticos como profilaxis antes de la cirugía reduce el riesgo de que el paciente desarrolle una infección del sitio quirúrgico. Esta investigación evidencia similares resultados en cuanto la incidencia de infecciones de herida quirúrgica, respecto a los factores es variable.

Finalmente, un estudio realizado por Fernández et al. (2016) también encontró evidencias parecidas en diversos hospitales de Cuba, hallándose que existe una frecuencia importante de infecciones quirúrgicas; además, entre los factores de riesgo inherentes a la intervención y/o técnica quirúrgica más relevantes figuran: horario en que se realiza la operación, tipo de cirugía (electiva o urgente), grado de contaminación de la intervención, quirófano donde se efectúa, técnica quirúrgica empleada, tiempo quirúrgico, utilización de transfusiones de sangre.

Como se puede ver, los resultados encontrados en el presente estudio coinciden con los estudios consultados tanto en el contexto nacional e internacional en cuanto a la frecuencia de infecciones quirúrgicas y sus factores relacionados. Es preocupante encontrar elevados índices de infecciones quirúrgicas puesto que esta podría generar daños secundarios en los pacientes. La evidencia encontrada es concordante con la frecuencia de infecciones y sus factores asociados encontrados en el presente estudio donde trabajo con historias clínicas de pacientes postoperados. En ese sentido sería importante realizar estudios prospectivos a fin de consolidar el hallazgo de factores asociados a infecciones de herida quirúrgica para tomar decisiones de control para estos problemas de salud.

En ese sentido Medina et al. (2011) afirma que en la actualidad las infecciones de herida quirúrgica continúan siendo uno de los eventos más frecuentes dentro de las complicaciones graves de una cirugía. Asimismo, Bernal (2014) señala que pese a las acciones sanitarias propuestas por la OMS y el MINSA, las infecciones no han disminuido su incidencia. Por tanto, es importante abordar de manera continua estos problemas mediante acciones dirigidas a controlar dichas infecciones.

Por tanto, Falci et al. (2011) enfatiza que el conocimiento sobre la infección quirúrgica y sus factores en lugares específicos es fundamental para tomar acciones que permitan controlar estas infecciones y evitar daños en el paciente. Se puede señalar que los índices de infecciones son importantes indicadores de evaluación para la calidad del cuidado dado al paciente. Además, según Bernal (2014) el conocimiento de las causas suficientes, necesaria y de los factores de riesgo de este tipo infecciones, permite proponer intervenciones sanitarias que reduzcan los indicadores de infecciones hospitalarias.

Al realizar la presente discusión sobre los resultados encontrados y los estudios revisados, así como la bibliografía sobre las infecciones quirúrgicas, se puede observar una incidencia importante de este tipo de infecciones. Además, se visualiza que en su mayor parte los factores asociados tienen que ver con condiciones relacionadas a la atención hospitalaria tales como son la duración prolongada de la cirugía y 
el rasurado de la zona operatoria. También existentes factores internos de las personas.

Siendo las infecciones operatorias un problema frecuente que está relacionado con la condición de atención hospitalaria, es importante realizar más investigaciones específicas a nivel local para conocer con mayor precisión los factores relacionados con las infecciones de herida quirúrgica, asimismo proponer protocolos y medidas de control basadas en evidencia científica. Por ello el presente trabajo pretende contribuir con esta discusión y análisis para conocer mejor la frecuencia de estas infecciones.

Cabe mencionar que el presente estudio evidenció la problemática de las infecciones quirúrgicas a nivel de un hospital local, donde hay poca o nula investigación de estos aspectos. Si bien es cierto los resultados de este estudio serían discutibles por tratarse de una investigación retrospectiva, pero existen varios estudios observacionales y prospectivos que evidencian similares resultados. Sin embargo, es importante que se realicen investigaciones a nivel local para fortalecer una cirugía sin riesgo para el paciente.

\section{CONCLUSIONES}

Existe una alta frecuencia importante de infecciones de herida quirúrgica $(35.3 \%)$ en los pacientes del Servicio de Cirugía, Hospital II-1 Moyobamba.

La apendicectomía fue la intervención quirúrgica que más se realizó $(76 \%)$ durante el tiempo de estudio, en el Hospital II-1 Moyobamba.

Las infecciones quirúrgicas fueron más frecuentes en pacientes de 18 a 23 años, de sexo femenino.

Las infecciones quirúrgicas fueron más frecuentes en pacientes con una intervención de tiempo prolongado, cuya zona operatoria fue rasurada.

Las condiciones que influyeron para una mayor frecuencia de infecciones quirúrgicas en los pacientes fueron la duración prolongada de la cirugía y el rasurado de la zona operatoria $(\mathrm{p}<0.05)$.

Las condiciones que no influyeron fueron: la edad, sexo, tipo de intervención, diabetes, obesidad y profilaxis antibiótica no dada $(\mathrm{p}>0.05)$.

\section{REFERENCIAS BIBLIOGRÁFICAS}

Alvia, M. (2013). Factores de riesgo asociados a la infección de sitio operatorio en pacientes post operados por apendicetomía convencional en el Hospital San José del Callao-Perú durante el periodo enero- diciembre 2012. Tesis para optar el Título de Médico Cirujano. Facultad de Medicina Humana, Universidad Ricardo
Palma. Lima.

Ángeles, U. (2014). Factores de riesgo relacionados con infección del sitio quirúrgico en cirugía electiva. Revista Cirugía y Cirujanos Volumen 82, No. 1, Enero-Febrero 2014. México.

Bernal, P. (2014). Factores de riesgo asociados a la infección de heridas quirúrgicas en pacientes del Servicio de Cirugía A del Hospital Regional del Cusco. Tesis para optar el Título Profesional de Médico Cirujano. Cusco: Facultad de Ciencias de la Salud, Universidad Andina del Cusco.

Canales et al. (1994). Metodología de la Investigación Cientifica: Manual para el desarrollo de personal de salud. México: Editorial Limusa.

Carbajal, R. \& Londoño, A. (2012). Factores de riesgo e infección del sitio quirúrgico en procedimientos de cirugía ortopédica con prótesis. Revista Chilena Infectol 2012; 29 (4): 395-400. Colombia; 2011.

Casado, D. (2014). Factores de riesgo de infección de la herida quirúrgica en cirugía electiva de cáncer de sigma y recto. Implantación de un programa de vigilancia. Tesis doctoral. Universidad de Valencia; España.

De la Cruz, J. (2015). Factores de riesgo para infección de sitio operatorio en pacientes postapendicectomia atendidos en el Hospital de Ventanilla año 2015. Tesis para optar el Título profesional de Médico Cirujano. Universidad Ricardo Palma. Lima; Perú.

Falci, F. et al. (2011). Riesgo para infección de sitio quirúrgico en pacientes sometidos a cirugías ortopédicas. Rev. Latino-Am. Enfermagem. Brasil.

Fernández, O. et al. (2016). Factores de riesgo relacionados con las infecciones posoperatorias. Revista MEDISAN 2016; 20(2):133. Cuba.

Hernández R. (2008). Metodología de la Investigación. 3ra edición; México: Editorial Mc. Graw Hill interamericana.

Kirby J. (2018). Prevención de las infecciones de la herida operatoria. Recuperado de: http://cirugiahcg.com.

Medina et al. (2011). Infecciones de las heridas quirúrgicas relacionadas a la atención perioperatoria. Revista Ciencia y Cuidado. España. 
Méndez, J. (2018). Limpieza y desinfección de material quirúrgico. Recuperado de: http://www.enfermeraspabellonyesterilizacion .cl/trabajos/material.pdf.

MINSA. (2000). Análisis de situación de las infecciones intrahospitalarias en Perú 1999 2000. Oficina General de Epidemiologia - Red Nacional de Epidemiología. Lima, Perú.

Richter L. (2016). Factores de riesgo asociados a infección de herida operatoria post cesárea en el Hospital Vitarte, periodo 2013 a junio 2015. Tesis para optar el Título de Médico Cirujano. Facultad de Medicina Humana, Universidad Ricardo Palma. Lima.

Rodríguez R. et al. (2017). Incidencia y factores de riesgo para infección de sitio quirúrgico en cirugias generales. Rev. Latino-Am. Enfermagem 2017; 25: e 2848. Brasil.

Supo J. (2014). Metodología de la investigación científica para investigación de la ciencia de la salud. $2^{\circ}$ edición. Lima: Editorial Moderna. Perú.

Tite S. (2012). Estudio de factores de riesgo que influyen en la infección nosocomial de heridas quirúrgicas de pacientes en el hospital provincial docente Alfredo Noboa Montenegro durante el periodo de enero a julio". Ambato Ecuador.

Vásquez M. et al. (2016). ¿Qué nos dicen los estudios de incidencia de infección de la herida quirúrgica?. Rev. Enfermería Investiga, Investigación, Vinculación, Docencia y Gestión-Vol. 3 Sup.1 2018. Ecuador. 